\title{
Percepción de los Beneficios de la Tenencia de Animales de Compañía para las Personas de la M ediana Edad con Diabetes Mellitus Tipo 2
}

\author{
Perceived Benefits of Pet Ownership by Middle-Aged People with Type 2 \\ Diabetes Mellitus
}

\author{
Beatriz Hugues H. ${ }^{1,2}$, Aimee M. Álvarez Á. ${ }^{1}$, Lizet Castelo E. ${ }^{1}$, Loraine Ledón L. ${ }^{1}$, \\ Madelín Mendoza T. ${ }^{1}$, Emma Domínguez A. ${ }^{1}$
}

\section{Resumen}

Se efectuó un estudio con el objetivo de explorar la percepción de personas de la mediana edad con Diabetes Mellitus Tipo 2 (DM2) respecto a la tenencia de animales de compañía y su relación con la satisfacción personal, beneficios sobre la salud psicoemocional, el control metabólico y la motivación para la realización de actividades físicas. El estudio fue de tipo transversal, descriptivo, con 85 personas de 40 a 59 años de edad con DM2 y residentes en La Habana, Cuba. Se confeccionó un cuestionario autoadministrado para la evaluación de estos aspectos y obtención de datos demográficos. El 82\% de las personas admitió que se sentían muy satisfechas al compartir sus vidas con estos animales, el $84 \%$ percibió una relación muy beneficiosa para su salud psicoemocional y el $98 \%$ admitió que los motivaba a cuidarse más de la DM2. Asimismo, dentro de las 43 personas que tenían perros, el $65 \%$ se sentía motivado a realizar actividades físicas junto al animal (caminatas).

Palabras clave: satisfacción personal, salud psico-emocional, control metabólico, actividades físicas, animales de compañía

\section{Abstract}

The study was conducted to explore the perception of people of middle-age with Type 2 Diabetes Mellitus (DM2) regarding ownership of companion animals and their relationship with personal satisfaction, benefits on mental health and metabolic control, and to the motivation on practicing physical activities. The study was descriptive, crosssectional, with 85 people from 49 to 58 years of age who had DM2, and were residents of Havana, Cuba. A self-administered questionnaire for assessing these aspects and

\footnotetext{
${ }^{1}$ Instituto Nacional de Endocrinología (INEN), La Habana, Cuba

${ }^{2}$ Clínica Central Cira García, La Habana, Cuba

${ }^{3}$ E-mail: bettymig@infomed.sld.cu
}

Recibido: 26 de julio de 2015

Aceptado para publicación: 20 de noviembre de 2015 
obtaining demographic data was constructed. The results showed that $82 \%$ of the people felt very satisfied to share their lives with companion animals, $84 \%$ perceived a very beneficial relationship for their mental health, and $98 \%$ admitted that they were motivated to take better care of DM2. Also, within the 43 people who have dogs, $65 \%$ felt motivated to perform physical activities with their animals (long walks).

Key words: personal satisfaction, psycho-emotional health, metabolic control, physical activities, companion animals

\section{INTRODUCCIÓN}

La convivencia con animales de compañía reporta beneficios para la salud física, psicológica y social de los seres humanos al facilitar un mejor desempeño social, mayor autoestima, sensación de bienestar, recuperación más rápida de sus problemas mentales o físicos (enfermedades cardiovasculares, metabólicas), reducción del sentimiento de soledad y de la depresión, desarrollo de la responsabilidad y del respeto hacia otras formas de vida (Medina, 2011); asimismo, promueven la relajación, recreación, distracción y la realización de actividades físicas como las caminatas (Jofré, 2005).

La diabetes mellitus tipo 2 (DM2) es una enfermedad crónica no transmisible, de alta incidencia en la población cubana (Bustillo et al., 2013). Los pacientes con DM2 pueden llegar a padecer alteraciones de la salud física relacionadas con el sistema cardiovascular (oscilaciones de la frecuencia cardíaca y la tensión arterial), de la salud mental (ansiedad, depresión, irritabilidad, estrés psicológico) y psicosocial (manifestar una pobre comunicación social dentro y fuera del marco familiar) (Pereira, 2009; Ledón, 2012).

En particular, las personas con DM2 de la mediana edad pueden experimentar sentimientos de indefensión y vulnerabilidades relacionadas con las manifestaciones psicológicas y físicas asociadas con los cambios resultantes de esta etapa de la vida como el envejecimiento, la emancipación de los hijos, la inclusión de nuevos miembros en la familia y la jubilación, lo que puede incidir negativamente en sus estados psíquicos y conducirlos al descontrol metabólico (Pérez, 2004; Alfonso, 2007).

Los animales de compañía pueden ser una fuente clave de respaldo social y emocional para sus dueños, ya sean personas 'comunes' o las que se enfrentan a problemas graves de salud, según se confirmó en un estudio desarrollado por McConnell et al. (2011) en Ohio, quienes hallaron que este tipo de personas eran más felices, tenían una mejor salud, una mayor autoestima, mejor aptitud física, tendían a sentirse menos solitarios, eran más concienzudos, más extrovertidos, tendían a ser menos temerosos y a estar menos preocupados que aquellas personas que no las tenían. No obstante, las autoras del presente estudio no han encontrado referencias sobre estudios que exploren los beneficios de la tenencia de animales de compañía en personas con DM2.

Sobre estas bases y teniendo en consideración los múltiples efectos positivos que podría brindar la tenencia de animales de compañía para las personas de la mediana edad con DM2, se hizo el presente estudio con el objetivo de explorar la percepción que tienen este tipo de personas residentes de La Habana, Cuba, con relación a la salud física y psicoemocional, al control metabólico y la realización de actividades físicas. 


\section{MaTeRIALES Y Métodos}

Se realizó un estudio descriptivo y transversal, en el que se utilizó metodología cuantitativa de investigación, durante los meses de enero a junio de 2014.

La población objeto de estudio quedó conformada por 85 personas de la mediana edad (40-59 años), sin distinción de sexos, con DM2, y que eran responsables de animales de compañía. Estas personas acudían con sus animales a la clínica principal de animales afectivos de La Habana «José Luis Callejas», para solicitar el servicio de la consulta de oftalmología. El conocimiento del diagnóstico de la DM2 fue el resultado de la propia referencia. Como criterio de exclusión se consideró a estas personas que presentaron condiciones físicas o de salud mental que dificultaran la lectura y comprensión del instrumento de recolección de la información utilizado.

El instrumento consistió en un cuestionario autoadministrado sobre la tenencia de animales de compañía, elaborado a los fines del estudio, a partir del cual se exploraron los siguientes aspectos:

Datos demográficos de las personas: sexo, edad, municipio de residencia, nivel de escolaridad, ocupación. Se registraba si convivían o no con otras personas.

Satisfacción personal sobre la tenencia de animales de compañía: Valoración de la satisfacción que siente la persona al estar en contacto directo con un animal de compañía, así como el bienestar que le produce esta interrelación. Se exploró sobre la especie de animal que poseían y el tiempo de convivencia con los mismos; además, se indagó sobre el animal preferido. Los encuestados podían seleccionar más de una respuesta. Beneficios sobre la salud psico-emocional: Valoración de los posibles efectos beneficiosos que ejerce la tenencia de animales de compañía, relacionados con los aspectos de la salud psicoemocional y la calidad de vida de las personas a su cargo.

- Beneficios sobre el control de la DM2: Valoración de los posibles efectos beneficiosos que ejerce la tenencia de animales de compañía sobre el control de la enfermedad de las personas que los atienden. El encuestado podía responder varias opciones en la pregunta sobre el efecto del tratamiento.

- Motivaciones para la realización de actividad física: Se entendieron como tales las razones que impulsaron la realización de actividades físicas (caminatas) y su frecuencia en relación con la tenencia de los animales de compañía. El encuestado podía elegir más de una respuesta en la pregunta sobre el efecto positivo de las caminatas.

Opinión sobre el instrumento utilizado y el tema en cuestión: Valoración de los aspectos referidos por los encuestados de forma espontánea, sobre el instrumento utilizado y la temática referida a los beneficios de la tenencia de animales de compañía.

La participación de los sujetos en el estudio fue voluntaria y anónima. A cada persona que cumplió con los criterios de inclusión se le solicitó su participación en el estudio, previa información de las características del mismo. Al consentir su participación se le brindó el cuestionario. Toda la información recogida se manejó de forma confidencial. No se recolectaron datos de identidad personal y los datos obtenidos fueron codificados y analizados mediante estadística descriptiva. Los resultados se expresaron en números absolutos.

\section{Resultados y Discusión}

\section{Datos Demográficos}

En el Cuadro 1 se presentan los resultados de los datos demográficos de las personas encuestadas. Se observó una mayor 
Cuadro 1. Datos demográficos de personas de mediana edad $(n=85)$ con Diabetes Mellitus Tipo 2 y tenencia de animales de compañía. La Habana, Cuba (2014)

\begin{tabular}{lcc}
\hline \multirow{2}{*}{ Variable } & \multicolumn{2}{c}{ Personas } \\
\cline { 2 - 3 } & N. & $\%$ \\
\hline Género & & \\
Mujeres & 61 & 72 \\
Hombres & 24 & 28 \\
Edad (años) & & \\
40-49 & 49 & 58 \\
50-59 & 36 & 42 \\
Residencia & & \\
Plaza de la & 38 & 45 \\
Revolución & 27 & 32 \\
Centro Habana & 13 & 15 \\
Habana Vieja & 7 & 8 \\
Otros & & \\
Nivel de escolaridad & 8 & 9 \\
Primaria & 7 & 8 \\
Secundaria & 36 & 43 \\
Medio & 34 & 40 \\
Superior & & \\
Ocupación laboral & 75 & 88 \\
Vinculados & 10 & 12 \\
No vinculados & & \\
Convivencia con otras & & \\
personas & 66 & 78 \\
Sí & 19 & 22 \\
No & & \\
\hline
\end{tabular}

presencia de mujeres $(72 \%)$. Las personas procedían de diversos municipios, principalmente de la Plaza de la Revolución (45\%) y Centro Habana (32\%). Si bien la clínica en la que se realizó el estudio es de referencia nacional, la mayor proporción de pacientes provino de los municipios cercanos. El nivel de escolaridad fue notoriamente mayor en el nivel medio preuniversitario $(43 \%)$ y universitario (40\%), donde el $88 \%$ de las personas tenía trabajo, hecho esperado dada la edad de las personas. Asimismo, fue mayoritario el número de personas que convivían con otros familiares $(78 \%)$ con respecto a las que vivían solas con sus animales.
Hugues y Torres (2014) realizaron estudios en estas mismas condiciones pero en personas mayores de 18 años y hallaron resultados similares en lo referente al subgrupo de la mediana edad. Por otro lado, en un estudio similar en Santa Clara, Cuba (Beovides et al., 2011), determinaron que la población estaba compuesta mayoritariamente por hombres (53.3\%), con edades entre 18 a 30 años $(42.7 \%)$ y de 31 a 50 años $(26.7 \%)$ y, especialmente, con un nivel de escolaridad medio (59.3\%). Esta información difiere en relación al género y la edad con los datos del presente estudio, pero concuerda con la mayor frecuencia respecto al nivel de escolaridad.

\section{Satisfacción Personal}

Los resultados de la evaluación de la satisfacción personal con respecto a la tenencia de animales de compañía se encuentran en el Cuadro 2.

La mayoría de los encuestados tenían perros $(51 \%)$, seguido de gatos $(21 \%)$ y aves ornamentales (9\%). El 9\% convivía con más de una especie, entre las que siempre se encontraba el perro. Asimismo, indicaron como animal preferido al perro (50\%), seguido por el gato $(23 \%)$ y las aves ornamentales $(16 \%)$, aunque no siempre hubo coincidencia entre el animal que poseían y el que preferían. Estos resultados coinciden con otros estudios cubanos realizados en otras ciudades del país (Beovides et al., 2011; Domínguez et al., 2011; Hugues y Torres, 2014).

Los resultados muestran que la mitad de los encuestados poseían sus animales por 10 o más años. Asimismo, el $82 \%$ concordaba en que estaban «muy satisfechos» $(82 \%)$ $\mathrm{o}$ «satisfechos» (18\%) al compartir sus vidas con estos animales. Ninguna persona mostró insatisfacción.

Al considerar la utilidad que representó la interrelación entre dueños y animales de compañía, las respuestas expresadas con mayor frecuencia se refirieron a que los animales les brindaban compañía (98\%), eran 
Cuadro 2. Evaluación de la satisfacción personal en relación con la tenencia de animales de compañía en personas de mediana edad con Diabetes Mellitus Tipo 2 (n=85) (La Habana, Cuba, 2014)

\begin{tabular}{|c|c|c|}
\hline \multirow{2}{*}{ Variable } & \multicolumn{2}{|c|}{ Personas } \\
\hline & N. ${ }^{\circ}$ & $\%$ \\
\hline \multicolumn{3}{|l|}{ Especie } \\
\hline Perros & 43 & 51 \\
\hline Gatos & 18 & 21 \\
\hline Aves & 8 & 9 \\
\hline Tortugas & 3 & 4 \\
\hline Roedores & 5 & 6 \\
\hline Otros & -- & -- \\
\hline Varios & 8 & 9 \\
\hline \multicolumn{3}{|c|}{ Tiempo de tenencia (años) } \\
\hline$<1$ & -- & -- \\
\hline $1-3$ & 5 & 6 \\
\hline $4-6$ & 10 & 12 \\
\hline $7-9$ & 28 & 33 \\
\hline$>9$ & 42 & 49 \\
\hline \multicolumn{3}{|c|}{ Satisfacción de la tenencia } \\
\hline Muy satisfecho & 70 & 82 \\
\hline Satisfecho & 15 & 18 \\
\hline \multicolumn{3}{|l|}{ Relación con el animal } \\
\hline Brinda compañía & 83 & 98 \\
\hline $\begin{array}{l}\text { Cuido mejor de mi } \\
\text { salud }\end{array}$ & 82 & 96 \\
\hline Brinda bienestar & 77 & 91 \\
\hline Libera tensiones & 75 & 88 \\
\hline $\begin{array}{l}\text { Compañero para mi } \\
\text { soledad }\end{array}$ & 72 & 85 \\
\hline Satisface contacto & 49 & 58 \\
\hline Mejora mi ánimo & 68 & 80 \\
\hline $\begin{array}{l}\text { Me comunico con él } \\
\text { Medio de }\end{array}$ & 60 & 71 \\
\hline $\begin{array}{l}\text { comunicación } \\
\text { personal }\end{array}$ & 65 & 76 \\
\hline
\end{tabular}

un estímulo para cuidarse más $(96 \%)$ y les brindaban bienestar (91\%). También expresaron que los liberaban de las tensiones $(88 \%)$ y que eran compañeros para los momentos de soledad (85\%). Resultados similares fueron obtenidos por Castillo y López (2013) en Tarragona, España.

\section{Beneficios sobre la Salud Mental}

El Cuadro 3 resume la percepción de las personas con relación a los beneficios de los animales de compañía sobre la salud psicoemocional. El 84\% expresó que les resultaba muy beneficiosa, en tanto que el 16\% indicó que era beneficiosa. Mocoso (2009) cita que este tipo de relación es «un mutuo beneficio y una relación dinámica entre las personas y otros animales que es influenciada por comportamientos que son esenciales para la salud y el bienestar de ambos».

El 79\% de personas recomendó la convivencia con animales de compañía, mientras que el $9 \%$ que no asintió indicó que se debería tener en cuenta los conocimientos sobre su crianza y las condiciones de vida que les permitan satisfacer sus necesidades, así como considerar las preferencias y gustos de cada individuo. Chur-Hansen et al. (2008) identificaron en un estudio sobre personas mayores que las razones para no tener animales de compañía eran mayormente de índole emocional (la muerte del animal, su desam-

Cuadro 3. Evaluación del beneficio de la relación de personas de mediana edad con Diabetes Mellitus Tipo 2 con sus animales de compañía ( $\mathrm{n}=85) \quad(\mathrm{La}$ Habana, Cuba, 2014)

\begin{tabular}{lcc}
\hline \multirow{2}{*}{ Variable } & \multicolumn{2}{c}{ Personas } \\
\cline { 2 - 3 } & N. ${ }^{\circ}$ & $\%$ \\
\hline Beneficios en la & & \\
relación personal & & \\
Muy beneficiosa & 71 & 84 \\
$\quad$ Beneficiosa & 14 & 16 \\
Recomienda la tenencia de & \\
animales de compañía & & \\
Sí & 67 & 79 \\
No & 8 & 9 \\
No sé & 10 & 12 \\
Mejora de la calidad de vida & \\
Sí & 73 & 86 \\
No & 7 & 8 \\
No sé & 5 & 6 \\
\hline
\end{tabular}


Cuadro 4. Evaluación de los beneficios de la tenencia de animales de compañía sobre el control de la diabetes mellitus tipo 2 (n=85) (La Habana, Cuba, 2015)

\begin{tabular}{ll}
\hline \multirow{2}{*}{ Variable } & \multicolumn{2}{c}{ Personas } \\
\cline { 2 - 2 } & $\mathrm{N} .{ }^{\circ} \%$ \\
\hline
\end{tabular}

En el cumplimiento

del tratamiento ${ }^{1}$

$\begin{array}{lcc}\text { Me cuido más } & 83 & 98 \\ \text { Motiva a caminar } & 44 & 52 \\ \text { Motiva a cuidar la } & 26 & 31 \\ \text { nutrición } & 5 & 6\end{array}$

Advierte cambio de conducta por aumento de glicemia ${ }^{2}$

$\begin{array}{lcc}\text { Sí } & 46 & 76 \\ \text { No } & 10 & 16 \\ \text { No lo nota } & 5 & 8\end{array}$

Toma cuidado al observar c ambio de conducta en el animal ${ }^{3}$

$\begin{array}{lcc}\text { Sí } & 39 & 85 \\ \text { No } & 7 & 15\end{array}$

Ayuda en el control de

la enfermedad

\begin{tabular}{lcc} 
Sí & 58 & 68 \\
No & 20 & 24 \\
No sé & 7 & 8 \\
\hline
\end{tabular}

${ }^{1}$ Puede haber más de una respuesta por encuestado

${ }^{2}$ Tenedores de perros y gatos $(n=61$ )

3 Aquellos que observaron cambios de conducta

paro si el dueño fallecía, y el hecho de tener familiares y no necesitar la compañía del animal) o pragmáticas (por no poder dedicarles tiempo, entre otras). Estos resultados implican que no se puede generalizar la recomendación de compartir la vida con animales de compañía.

El 86\% del grupo encuestado respondió de forma afirmativa al indagar si la convivencia con esta clase de animales aportaba calidad a sus vidas. Por otro lado, Alabama Cooperative Extension System (2011) comu- nicó que la vida en compañía de perros ha demostrado proveer beneficios (fisiológicos y psicológicos) que pueden mejorar la calidad de vida de las personas.

\section{Beneficios sobre el Control de la Diabe- tes}

Al analizar la motivación del cumplimiento del tratamiento de la DM2 al convivir con un animal de compañía (Cuadro 4), se observó que casi la totalidad (98\%) de los sujetos del estudio estaban motivados por cuidarse más. Las razones que exponían estaban relacionadas sobre todo con el hecho de que su animal podía quedar desvalido o desprotegido en caso de discapacidad o muerte, siendo así que los animales constituían un foco de atención y preocupación.

Al preguntarle a las personas poseedoras de perros y gatos $(n=61)$ si observaban cambios de conducta en su animal ante episodios de descontroles de la glucemia (temblores, fatigas, otros), el $76 \%$ respondió en forma positiva, ya que sus animales se ponían nerviosos, ladraban o maullaban, o permanecían más atentos de lo acostumbrado, sin separarse de ellos. El 85\% (39/46) de las personas que habían advertido los cambios en la conducta de sus animales indicaron que este comportamiento les motivaba a tomar mayores cuidados, tratando de ajustar su tratamiento para evitar daños y complicaciones de su salud y no dejar desvalidos o desamparados a sus animales. Asimismo, el 68\% de los encuestados indicó que la tenencia de animales de compañía contribuía de manera beneficiosa en el control de la DM2.

Wells et al. (2008) encontraron cambios conductuales en los perros al advertir episodios de hipoglucemia en sus dueños; asimismo, los dueños de gatos declararon que estos se mostraban muy atentos ante la presentación de signos de hipoglucemia en ellos; asimismo, se ponían nerviosos e inquietos y no se separaban de su lado. Estos resultados sugieren que la tenencia de perros y gatos podría ser de gran utilidad en personas con DM2, tal y como lo plantea Martínez (2013). 


\section{Motivaciones para la Actividad Física}

En el análisis sobre la motivación por la realización de actividades físicas, solo se tuvieron en cuenta las personas que tenían perros $(n=43)$ (Cuadro 5). En este grupo, el 65\%

Cuadro 5. Evaluación de la motivación de la actividad física en personas de mediana edad con Diabetes Mellitus Tipo 2 en relación con la tenencia de perros como animales de compañía ( $\mathrm{n}=43$ ) (La Habana, Cuba, 2014)

\begin{tabular}{|c|c|c|}
\hline \multirow{2}{*}{ Variable } & \multicolumn{2}{|c|}{ Personas } \\
\hline & $\mathrm{N} .^{\circ}$ & $\%$ \\
\hline \multicolumn{3}{|l|}{ Realiza caminatas } \\
\hline Sí & 28 & 65 \\
\hline No & 15 & 35 \\
\hline \multicolumn{3}{|l|}{ No realiza caminatas } \\
\hline Limitación física & 4 & 27 \\
\hline $\begin{array}{l}\text { No le gusta salir con el } \\
\text { perro }\end{array}$ & 3 & 20 \\
\hline Peligro para el perro & 12 & 80 \\
\hline Peligro para la persona & 4 & 27 \\
\hline $\begin{array}{l}\text { Prefiere que otros lo } \\
\text { paseen }\end{array}$ & 5 & 33 \\
\hline \multicolumn{3}{|l|}{ Sí realiza caminatas } \\
\hline $1-2 /$ mes & -- & -- \\
\hline $1 /$ semana & -- & -- \\
\hline 2-3/semana & 3 & 11 \\
\hline$>3$ semana & 11 & 39 \\
\hline Todos los días & 14 & 50 \\
\hline \multicolumn{3}{|l|}{ Tiempo de las caminatas } \\
\hline $15-30 \mathrm{~min}$ & 22 & 79 \\
\hline $31-60 \mathrm{~min}$ & 6 & 21 \\
\hline \multicolumn{3}{|l|}{ Efecto de las caminatas } \\
\hline Me mantiene saludable & 27 & 96 \\
\hline Disfruto de la compañía & 25 & 89 \\
\hline Promueve las caminatas & 25 & 89 \\
\hline Me produce relajación & 22 & 79 \\
\hline Estímulo para ejercitar & 17 & 61 \\
\hline $\begin{array}{l}\text { Ayuda al control } \\
\text { me tabólico }\end{array}$ & 12 & 43 \\
\hline Ayuda a bajar de peso & 11 & 39 \\
\hline Ejercicio para el perro & 6 & 21 \\
\hline Otros & -- & -- \\
\hline
\end{tabular}

realizaban caminatas con sus perros, especialmente por 15-30 minutos (79\%), predominando aquellos que lo hacían a diario $(50 \%)$.

La mayor motivación para la ejercitación física con estos animales estuvo referida a que los ayuda a mantenerse saludables (96\%), seguido del disfrute de la compañía del animal (89\%), de que les promueve realizar las caminatas $(89 \%)$ y del efecto relajante que les produce esta práctica $(79 \%)$. Asimismo, Cangelosi y Sorrell (2010), demostraron que las personas adultas que caminan con perros lo hacían de forma más constante que quienes lo hacían con otro ser humano como compañía.

Los sujetos que no caminaban con los animales $(n=15)$ alegaron que podía resultar peligroso para el animal (80\%) o para ellos mismos (27\%), esta última respuesta, coincidente con quienes refirieron tener limitaciones físicas (27\%). Asimismo, el 33\% prefería que sean otras personas los que saquen a pasear al perro.

Los resultados alcanzados indican el beneficio de la tenencia de animales de compañía en personas adultas, al constituir una vía de motivación para la realización de caminatas, favoreciendo la salud física y mental.

\section{Literatura Citada}

\section{Alabama Cooperative Extension} System. 2011. La compañía del perro y sus beneficios para el ser humano. Alabama A\&M and Auburn Universities. UNP-58. [Internet]. Disponible en: http://www.aces.edu/pubs/docs/ U/UNP-0058/UNP-0058.pdf

2. Alfonso JC. 2007. Cuba: características socio demográficas de las mujeres de edad mediana. En: Artiles L, Navarro D, Manzano B (eds). Climaterio y menopausia, un enfoque desde lo social. La Habana: Ed Científico Técnica. p 21-37. 
3. Beovides LM, Silveira PE, Norman MO. 2011. Evaluación del cuidado de la salud de mascotas en el municipio Santa Clara, Cuba. En: VII Congreso Internacional de Ciencias Veterinarias. La Habana, Cuba.

4. Bustillo EE, Bustillo EM, Pérez F, Pérez R, Brito A, González A, García Y. 2013. Prevalencia de la diabetes mellitus y de la glucemia alterada en ayunas en un área de la ciudad de Sancti Spíritus. Rev Cubana Endocrinol 24: 107114. [Internet]. Disponible en: http:// scielo prueba.sld.cu/scielo.php? script $=$ sci_arttext\&pid=S1561-29532013000 $200002 \& \operatorname{lng}=$ es\&nrm $=$ iso

5. Cangelosi P, Sorrell, J. 2010. Walking for therapy with man's best friend. J Psychosoc Nurs Ment Health Serv 48(3): 19-22. doi: 10.3928/02793695-20100202-05

6. Castillo A, López L. 2013. Animales, gran terapia a nivel emocional. Tesis de Enfermería. Tarragona, España: Universitat Rovira i Virgili. 28 p.

7. Chur-Hansen A, Winefield H, Beckwith M. 2008. Reasons given by elderly men and women for not owning a pet, and the implications for clinical practice and research. J Health Psychol 13: 988-995. doi: 10.1177/1359105 308097961

8. Domínguez H, Pérez Y, Barrios V. 2011. Características de la tenencia de la población felina en la ciudad de Matanzas. En: VII Congreso Internacional de Ciencias Veterinarias. La Habana, Cuba.

9. Hugues B, Torres M. 2014. Datos demográficos de animales de compañía atendidos en consulta de oftalmología y de las personas a su cargo. Rev Investig Vet Perú 25: 374-380. doi: 10.15381/ rivep.v25i3.10111

10. Jofré LM. 2005. Visita terapéutica de mascotas en hospitales. Rev Chil Infectol 22: 257-263. doi: 10.4067/S071610182 005000300007
11. Ledón L. 2012. Impacto psicosocial de la diabetes mellitus, experiencias, significados y respuestas a la enfermedad. Rev Cubana Endocrinol 23: 76-97. [Internet]. Disponible en: http:// scieloprueba.sld.cu/scielo.php? script $=$ sci_arttext $\&$ pid $=S 1561$ 29532012000100007\&lng=es\&nrm=iso

12. Martínez LE. 2013. Terapia asistida con animales. Un perro de asistencia para cada discapacidad. [Internet]. Disponible en: http://www.elcisne.org/noticia/unperro-asistencia-cada-discapacidad/ 2460.html

13. McConnellAR, Brown CM, Shoda TM, Stayton LE, Martin CE. 2011. Friends with benefits: on the positive consequences of pet ownership. J Pers Soc Psychol 101: 1239-1252. doi: 10.1037/a0024506

14. Medina C. 2011. La ética de la responsabilidad y el respecto a las mascotas como formas de vida - como solución al maltrato y abandono de las mismas. Tesis de Máster en Bioética. Bogotá, Colombia: Universidad El Bosque. 69 p.

15. Mocoso F. 2009. Caninoterapia para la atención de pacientes con evento cerebro vascular. Estudio piloto. Tesis de Maestría. Bogotá, Colombia: Universidad Sergio Arboleda. 94 p.

16. Pereira M. 2009. Link between psychopathology and quality of life in diabetic patients. In: XX World Diabetes Congress. Montreal, Canada.

17. Pérez V. 2004. Respuesta adaptativa a la diabetes mellitus y su relación con el envejecimiento. Rev Cubana Med Gen Integr 20(4). [Internet]. Disponible en: http://scielo.sld.cu/scielo. php? script= sci_arttext\&pid=S0864-21252004000 $400007 \& \operatorname{lng}=$ es $\& n r m=$ iso\&tlng $=e s$

18. Wells DL, Lawson SW, Siriwardena $N$. 2008. Canine responses to hypoglycemia in patients with type 1 diabetes. $\mathrm{J}$ Altern Complement Med 14: 1235-1241. doi: 10.1089/acm.2008.0288 